\title{
Inversión privada y pública en el desempeño de los sectores de la economía mexicana: 1993-2015*
}

\section{Private and public investment in the performance of the Mexican economy's sectors: 1993-2015}

\author{
Eduardo Ramírez-Cedillo** y Francisco López-Herrera***
}

\begin{abstract}
RESUMEN
Este artículo presenta los resultados del análisis de la influencia que tienen la inversión pública, la inversión privada y la tasa de interés sobre el desempeño de los sectores de la economía mexicana, tomando en cuenta los efectos tanto de largo como de corto plazo, así como la interacción entre la producción y la dinámica del desempeño de esos sectores. Mediante la estimación de modelos Autorregresivos de Rezagos Distribuidos (ARDL), se analiza el proceso de la dinámica de cada sector y su ajuste a la relación de equilibrio en el largo plazo, que fue detectada mediante pruebas convencionales para series de tiempo. Entre otros hallazgos, los resultados del análisis empírico muestran la existencia de debilidades estructurales en el sector primario, un alto grado de desvinculación del sector secundario y un sector terciario favorecido por la inversión pública y privada también como por la política de tasa de interés.
\end{abstract}

Palabras clave: Inversión pública, inversión privada, tasa de interés y desempeño económico, desempeño económico sectorial, economía mexicana.

Clasificación JEL: E22, E27, E69, H50, H54

\begin{abstract}
This paper shows the results of the analysis of the influence of the public investment, private investment and the interest rate on the performance of the Mexican economy's sectors, taking into account the long run and short run effects, as well as the interaction among the production and the performance's dynamics of those sectors. By means of the estimation of ARDL models, the process of the dynamics of each sector is analyzed so as their adjustment to the long run equilibrium relationship that was detected through conventional tests for time series. Among other findings, the results of the empirical analysis show the existence of structural weaknesses in the primary sector, a high degree of decoupling of the secondary sector, and a third sector favored by the public and private investment as well as the interest rate policy.
\end{abstract}

Keywords: Public investment, private investment, interest rate and economic performance, economic sectors performance, Mexican economy.

JEL Classification: E22, E27, E69, H50, H54

* Fecha de recepción: 20-01-2017. Fecha de aprobación: 20-02-2017

** Universidad Autónoma Metropolitana. Correo: ramceed@prodigy.net.mx. ORCID: 00000002-9524-3093

*** Universidad Nacional Autónoma de México. Correo: francisco_lopez_herrera@yahoo. com.mx. ORCID: 0000-0003-2626-9246 


\section{INTRODUCCIÓN}

En la literatura especializada se ha discutido de manera amplia la relación entre el gasto en inversión pública y privada, siendo ampliamente conocido el denominado efecto crowding out, que se refiere al desplazamiento de la inversión privada por la pública. También se reconoce la posibilidad de que se pueda presentar un efecto crowding in, lo que implicaría que la inversión pública propiciara, alentara o estimulara a la inversión privada.

Uno de los mecanismos de transmisión usuales para explicar el efecto del desplazamiento está relacionado con la variación de la tasa de interés. El modelo IS-LM supone que, ante el incremento del gasto gubernamental se podría tener por resultado un aumento de la tasa de interés que conduciría a menor inversión privada, siempre que la oferta monetaria permaneciera constante. En dicho modelo resulta importante el destino del gasto ya que el efecto esperado puede ser diferente si el gasto es de capital o de consumo.

Para el caso de México, Ramírez y López (2014) encuentran evidencia sobre una relación positiva entre la inversión privada y la pública tomando como referencia el periodo que va de 1993 a 2013, es decir, el periodo correspondiente a las reformas dirigidas a la apertura económica y financiera, consideradas acordes con el Consenso de Washington. En el trabajo antes mencionado, los autores llegan a resultados que permiten descartar la presencia de un efecto crowding out y aportan evidencias para establecer una retroalimentación entre la inversión pública y privada, además de que en ambos casos las variables se vinculan de forma positiva con el crecimiento de la economía.

Partiendo de un escenario similar al usado por los autores mencionados, en este trabajo se especifica un modelo econométrico que busca verificar los efectos que tienen la inversión pública, privada y la tasa de interés sobre el comportamiento de la economía, este estudio se enfoca en el nivel sectorial de actividad económica: primario, secundario y terciario. Dado que en dichos sectores se agrupan actividades distintas, es natural suponer que la dinámica de crecimiento en cada uno de ellos es diferente.

La composición de la actividad económica total en México se integra de manera preponderante con las actividades del sector terciario que en 1993 representaban 56 por ciento del total y que, 20 años después han pasado a más de 60 por ciento; en este periodo el sector terciario ha demostrado un mayor dinamismo, con una tasa de crecimiento anual promedio de 2.93 por ciento en todo el periodo; el sector primario creció en promedio a una tasa de 1.79 por ciento y el sector secundario a 2.14 por ciento. 
El cambio en la integración de la actividad económica en México no es privativa del país. La recomposición del Producto Interno Bruto (PIB) depende del incremento en el nivel de ingreso per cápita, por lo que se puede comprobar de forma empírica que entre más alto es el ingreso per cápita de un país, más representativo es el sector terciario. Según datos del Banco Mundial para el periodo de 2000 a 2012, se puede observar que los países clasificados como de ingresos bajos agrupan en su sector terciario un promedio cercano a 45 por ciento de su actividad total, mientras que los de ingresos medios, medios altos, altos y los de la Organización para la Cooperación y el Desarrollo Económicos (OCDE), agrupan 51, 59, 65 y 69 por ciento, respectivamente.

El objetivo de la primera parte de esta investigación es analizar la influencia que tiene la inversión pública y privada, así como la tasa de interés sobre el desempeño de los sectores en la economía mexicana para poder entender las características básicas de su dinamismo y, avanzar en la comprensión de su desempeño en el nivel de la actividad sectorial. La segunda parte se dedica a la revisión de la literatura pertinente, se describen los aspectos metodológicos del análisis y se muestran los resultados del análisis empírico, por último, hay una sección dedicada a la presentación de las conclusiones.

\section{REVISIÓN DE LA LITERATURA}

Durante mucho tiempo se ha pensado que la intervención del Estado en la economía es el origen de la inestabilidad monetaria, se cree que algunas de las fuentes de financiamiento usadas para mantener su gasto pueden ser la causa de constantes desequilibrios en los precios y en la tasa de interés. Cuando un gobierno se financia mediante la emisión monetaria, es altamente probable que esto resulte en el incremento de precios y, en el caso del uso de deuda como fuente de financiamiento, es posible que haya un aumento en la tasa de interés ${ }^{1}$. En ambos casos, se puede esperar un escenario negativo para la inversión privada y el desempeño económico.

La relación empírica entre la inversión privada y pública puede presentarse de tres formas: nula, complementaria o negativa. En el primer caso los cambios en la inversión pública no generan cambios en la privada; en el segundo, los cambios en la inversión pública generan cambios positivos en la inversión priva-

${ }^{1}$ Para algunos economistas independientemente de si el financiamiento se realiza por medio de la emisión de dinero o de bonos, la posibilidad de generar presiones inflacionarias siempre está presente ya que un país que ejerce déficit constante tarde o temprano tendrá que financiarlo mediante la emisión monetaria (Sargent y Wallace 1981; Catao y Terrones, 2003). 
da, a esa relación se le conoce como crowding in y se puede explicar a través de los efectos de derrama que tiene la inversión pública y que propician, alientan o impulsan a la privada; por ejemplo, la generación de infraestructura pública puede ser un detonador de inversión privada en la forma de diferentes esfuerzos individuales que tratan de aprovechar la nueva infraestructura. Cuando la relación es negativa, se le denomina crowding out, que es el desplazamiento de la inversión privada por la pública.

Fonseca (2009) considera las tres relaciones en un escenario, en el cual supone una política fiscal expansiva y una cantidad de dinero constante, esto tendrá como resultado un cambio en la composición del ingreso nacional, pero no en el nivel, lo que de forma inmediata genera la percepción de que dicha política fiscal expansiva es deficitaria y que presenta crowding out. Por su parte Haque y Kneller (2015) relacionan la inversión pública y privada de forma indirecta a través de la corrupción, exponen que en aquellos países donde los índices de corrupción son amplios, el rendimiento de la inversión pública es limitado, esto tiene efectos negativos sobre el crecimiento de la economía, mismos que se ven exacerbados por la caída de la inversión privada.

De forma teórica las posibilidades descritas con anterioridad se pueden agrupar en tres vertientes: la explicación del Teorema de la Equivalencia Ricardiana, consistente con la relación nula; el crowding in con la explicación keynesiana y el crowding out con la explicación neoclásica.

\section{I.1. Teorema de Equivalencia Ricardiana}

El Teorema de la Equivalencia Ricardiana (TER), parte de la idea esbozada por David Ricardo, retomada por Robert Barro $(1974 ; 1989 ; 1996)$ en años más recientes, este teorema considera que para financiar su gasto el gobierno siempre cuenta con dos medios de financiamiento: los impuestos y la deuda. Sin embargo, bajo ciertas consideraciones, no importa si el financiamiento del gasto se hace mediante una ampliación de impuestos o de deuda en el presente, ya que en el futuro los agentes económicos racionales esperarán, si se incrementó la deuda, un aumento en los impuestos. En este sentido se puede inferir que en el largo plazo el gobierno sólo cuenta con los impuestos como instrumento para el financiamiento del gasto público.

La implicación de fondo del TER consiste en que si el déficit presupuestal es financiado mediante la venta de bonos gubernamentales, no tendrá efectos en la demanda agregada de la economía, porque los hogares no aumentarán su consumo debido a la expectativa de que en el futuro se incrementen los impuestos, por lo 
tanto, la decisión de la venta de bonos es únicamente la de posponer el incremento en los impuestos.

Cuando se decide disminuir los impuestos y se instrumenta la emisión de bonos, en virtud de que el gasto del gobierno permanece constante, se supone que los agentes privados dedican parte de su ingreso disponible para adquirir los bonos. Ahora bien, la pregunta es si estos títulos han incrementado la riqueza neta de los poseedores. De acuerdo con Barro (1974), no existe un aumento en la riqueza neta porque el valor de adquisición de los bonos es igual al valor presente de los impuestos futuros ${ }^{2}$. Por lo tanto, no se registrarán aumentos en el gasto de consumo y la estrategia fiscal no incide positivamente sobre la demanda agregada.

Fuster (1993) presenta un estudio para cinco países europeos en los cuales, si bien no se comprueba el TER, la autora encuentra que los agentes económicos en cierta medida toman sus decisiones de acuerdo con las disposiciones de las finanzas públicas. Fonseca (2001), en una investigación para la economía portuguesa concluye que los resultados son ambiguos, por lo que no se puede verificar el efecto del TER por medio de la evidencia obtenida. En su estudio sobre Alemania, Lucke (1999) encuentra que aún cuando no existen pruebas contra el TER en dicho país, el modelo instrumentado rechaza enérgicamente esta postura. También se recomienda revisar Ricciuti (2003).

\section{I.2. Crowding in y Crowding out}

Un par de consideraciones cruciales para determinar el efecto que tendrá la inversión pública sobre la privada y, en última instancia, sobre el crecimiento de la economía tienen que ver con el tipo de postura fiscal que se adopte y la postura de la política monetaria. No se debe perder de vista que, en algunos casos, el tipo de gasto efectuado por el gobierno puede ser sustancial en las relaciones que se pueden esperar, ya que no toda inversión pública es necesariamente tan productiva como lo podrían ser, incluso, algunos gastos corrientes. Tomando en cuenta lo anterior, autores como Kneller et al. (1998) y Bleany et al. (2001), proponen una clasificación en la cual se separe el gasto público en gastos productivos y no productivos. La idea es retomada por Bayraktar y Moreno-Dobson (2015), quienes sugieren que

${ }^{2}$ Lasa $(1997,83)$, sostiene que "cuando se produce la rebaja de impuestos y se vende la deuda pública, la sociedad privada debería contabilizar un aumento de sus activos por la compra de los bonos y un aumento en sus pasivos intangibles correspondientes a los impuestos futuros, cuyo valor presente es igual al valor de los bonos. Por lo tanto, la disminución de impuestos y la emisión correspondiente de bonos no modifica en absoluto la riqueza neta de la sociedad". 
la relación entre el gasto público y el crecimiento depende de la selección del país donde se explore la relación y la clasificación del gasto, ya que, explican, la relación es significativa para aquellos países con estabilidad macroeconómica que son capaces de dirigir su gasto a propósitos productivos.

Si se instrumenta una postura fiscal expansiva deficitaria y una política monetaria restrictiva, el resultado esperado, por lo menos en el corto plazo, será un efecto desplazamiento. Es posible esperar en el largo plazo un efecto de complementariedad en función del tipo de inversión pública realizada.

No obstante, la aseveración convencional es puesta en duda, al menos para el caso de Estados Unidos, por Traum y Yang (2015), quienes usan un modelo neokeynesiano del tipo de equilibrio general dinámico estocástico para el periodo 1983:12008:1, estiman que el incremento de deuda no conlleva en el corto plazo un crowding out ya que la reducción de impuestos al capital y el incremento de la inversión pública podría estimular la inversión privada en lugar de desincentivarla. En el largo plazo el efecto dependerá de la distorsión generada por los impuestos usados para cubrir la deuda.

Por otra parte, el tipo de inversión pública realizada es de suma relevancia, sobre todo en economías en desarrollo, ya que, en algunos casos, el gasto destinado a este propósito no incrementa de forma automática el crecimiento de la economía (Pritchett, 2000). Un ejemplo se puede encontrar en Beteta y Moreno-Brid (2014; 72-73), quienes documentan un caso en el cual, aun cuando Honduras y Nicaragua presentan las mayores relaciones de Formación Bruta de Capital Fijo/PIB entre los países de Centroamérica y Jamaica, también presentan las menores tasas de crecimiento, esto se puede explicar por la ineficiencia de la inversión, menor productividad del trabajo, que la inversión se realiza en sectores de baja productividad, etcétera.

En caso que se experimente una política fiscal expansiva deficitaria con una política monetaria de tipo más acomodaticia es posible pensar en un crowding in de corto y de largo plazo ya que, como se ha mencionado por diferentes economistas (Eisner 1992; Vickrey, 2000), el impacto del gasto público sobre la tasa de interés es resultado de la respuesta que tenga el Banco Central sobre la cantidad adicional de gasto.

Teóricamente debería esperarse que cuando se presenta crowding in el impacto sobre la actividad económica debería de ser positivo, independientemente del orden de causalidad que pudiera suponerse, es decir, la presencia de un efecto feedback entre la inversión pública y privada. Evidencia del feedback es presentada por Afonso y Aubyn (2009) para 14 países de la UE, Canadá, Japón y Estados Unidos. Por otra parte, cuando la inversión pública afecta a la inversión privada de forma positiva, en todos los casos el efecto sobre el crecimiento es positivo, lo mismo sucede cuando la inversión privada es la que explica a la inversión pública. 
De acuerdo con el párrafo anterior, el crowding in supone de inmediato una relación positiva entre la inversión pública y el crecimiento económico ya que estimula la inversión privada. Existen diferentes canales de transmisión para el crowding in. De acuerdo con Annala et al. (2008), se puede mejorar la eficiencia de la producción al mejorar la infraestructura, por ejemplo, cuando se invierte en obras de suministro energético, mejora la eficiencia de la producción del trabajo en el sector privado. Cuando la inversión pública se canaliza en carreteras hay diferentes efectos positivos, como la ampliación de los mercados y mejores vías de acceso a los mismos, gracias a ello incrementan las ganancias y se generan posibilidades de instrumentar economías de escala; por otra parte también mejora la calidad de vida de las personas y su productividad al acortar los tiempos de traslado de las ciudades a la periferia. La potencialidad de los efectos positivos de la inversión pública se establece en su propia capacidad de alterar los precios relativos en la economía, por otra parte sus limitaciones radican en la forma en que se realiza su financiamiento.

El crowding in está asociado con el pensamiento keynesiano y también puede ser vinculado al desarrollo de Hirschman (1961), quien, bajo la idea de la necesidad de un crecimiento con desequilibrios inducidos con el objetivo de estimular la economía y la movilización de los recursos disponibles en la dirección correcta, supone una condición de "escasez" y "cuellos de botella" que, necesariamente, implican el impulso y fomento de nuevas inversiones públicas o privadas pues dicha condición sirve como mecanismo de inducción. Pese a la semejanza en los planteamientos de ambos autores, la diferencia podría ser el tipo de inversión que se necesita, en el caso de Keynes la subutilización de la capacidad productiva y niveles excesivamente bajos de inversión, explican el bajo nivel de ahorro, mientras que para Hirschman la insuficiencia de la capacidad productiva y del ahorro real se puede explicar por la escasa inversión de alta productividad (Posadas, 2008); por lo tanto no sólo es necesario el gasto público en inversión, sino una planificación acertada para determinar el tipo de proyectos de inversión que se realizarán. A decir de Hirschman $(1961 ; 18)$ "en una situación de escaso desarrollo se requiere un factor mucho más poderoso que el gasto deficitario u otro remedio keynesiano, utilizados con el fin de terminar con el desempleo". Para Erden et al. (2006), la inversión pública incentiva la inversión privada cuando mejora su productividad y compensa mejor su riesgo.

La infraestructura pública es un buen ejemplo de la manera en que se puede obtener crowding in ya que, es bien conocida la relación que tiene sobre el crecimiento económico. De acuerdo con Carranza et al. (2011), la caída en la inversión pública en América Latina es considerada con frecuencia como un factor que explica el bajo desempeño de la economía y el persistente nivel de inequidad y 
pobreza. Tal parece que en el tema de infraestructura la reducción del gasto público implica una reducción de la inversión privada, lo que en el sentido inverso podría no necesariamente ser cierto y tendría que ver con el tipo y calidad de inversión, además de un marco institucional adecuado que ofrezca certeza de la inversión pública y regule la inversión privada.

Lo cierto es que la consolidación presupuestal de varios países en América Latina en la década de los ochenta y noventa estuvo sustentada en la reducción de su gasto de inversión principalmente, se esperaba que la tasa de interés respondería de forma favorable al mejorar la sustentabilidad de las finanzas públicas y con ello la inversión privada ocuparía el lugar que quedaba vacante. De acuerdo con Fay y Morrison (2007) la participación privada no fue capaz de compensar el retroceso público, por lo que se estima necesario que se gaste más y mejor en infraestructura, incluyendo la realizada por el sector privado con participación central del Estado. Para el caso específico de México, Ramírez (2007) da cuenta de una reducción de la inversión total ante los ajustes a las finanzas públicas, sustentados básicamente en la reducción del gasto de capital durante el periodo 1980-2003.

Pereira (2000) realiza un interesante estudio para la economía de los Estados Unidos en el periodo 1956-1997 aplicando un modelo vectorial autorregresivo (VAR) en el que utiliza las variables correspondientes a la inversión privada, pública, PIB y el empleo privado, además de realizar un desglose de la inversión pública en cinco rubros descontando el gasto de inversión y actividades militares. Las rubros de inversión son: $a$ ) calles y carreteras; $b$ ) instalaciones eléctricas y de gas, sistemas de tránsito, aeropuertos, etcétera; $c$ ) sistemas de alcantarillado y suministro de agua; $d$ ) construcción de escuelas, hospitales, estaciones de policía, bomberos, salas de justicia, auditorios, terminales de pasajeros, estacionamientos, etcéte$\mathrm{ra} ; e$ ) conservación y desarrollo de estructuras correspondientes a acciones que tienen que ver con el agua, la tierra y la protección de animales, así como equipos civiles. Los resultados a nivel agregado indican un crowding in, positivo sobre la actividad económica $^{3}$ y un efecto menor sobre el empleo privado, la inversión pública desglosada arroja resultados similares con excepción de los rubros $a$ ) y $b$ ) que presentan un efecto negativo.

Hamed y Miller (2000), hacen también una desagregación de la totalidad del gasto público para 39 países en un periodo que va de 1975 a1984, por medio de un par de simulaciones con dos fuentes diferentes de financiamiento, impuestos y deuda. Las conclusiones a las que llegan los autores se pueden resumir de la siguiente manera: existe una diferencia en los efectos que se tienen en función del

${ }^{3}$ Como resultado de la investigación se estima que en el largo plazo 1 dólar gastado en inversión pública incrementa la actividad económica en 4.46 dólares. 
nivel de desarrollo del país, el financiamiento por impuestos en general expulsa a la inversión más frecuentemente que el financiamiento mediante gasto, el gasto público destinado a seguridad social y bienestar, independientemente del tipo de financiamiento presenta crowding out.

Es posible pensar que, cuando se toma el gasto total del gobierno sin realizar alguna separación relacionada a la inversión pública tradicional o bien en un escenario más amplio al gasto productivo, sobre todo en el caso de los países en desarrollo, el resultado sea el crowding out. Furceri y Sousa (2011) presentan evidencia respecto a dicho efecto, usan un modelo de datos panel para 145 países en el periodo de 1960-2007, la particularidad es que en dicho modelo se evalúa el gasto de gobierno únicamente sobre el gasto de consumo privado.

$\mathrm{Al}$ analizar el crowding out hay dos posibilidades interesantes que amplían su estudio, en principio la inversión pública puede desplazar a la inversión privada y mantener una condición de expansión económica, o bien el efecto desplazamiento puede ser de orden "fuerte", tanto así que el incremento de la inversión pública pueda incidir en una contracción del producto. Afonso y Aubyn (2009), encuentran evidencia de estas dos posibilidades en un estudio realizado en 17 países en el periodo de 1960-2005, en el cual aun cuando países como Italia, Japón, Francia y Estados Unidos habían presentado un efecto desplazamiento no se había tenido una afectación tan fuerte en su desempeño económico. De acuerdo con los autores, en los casos en los que la inversión privada desplazó a la pública, el impacto sobre la actividad económica siempre fue positivo.

En un panel de 95 países desarrollados y en vías de desarrollo para un periodo de estudio de 1970-2008, al desglosar los componentes fiscales, Afonso y Tovar (2015) encuentran una serie de efectos diferenciados sobre la inversión pública y privada. En principio el pago de intereses y las transferencias tienen efectos negativos sobre la inversión pública y privada, por otro lado, el gasto en seguridad social también tiene efectos negativos sobre la inversión privada, mientras que el gasto en temas de salud la favorece. En el caso de los subsidios a ciertas actividades como es la I+D se pensaba que estos tenían un efecto de crowding out para la inversión privada, sin embargo, la nueva evidencia, de acuerdo con Becker (2015), sugiere que políticas públicas de subsidios tienen efectos positivos para la inversión privada en $\mathrm{I}+\mathrm{D}$

Hablando de crowding in y crowding out para el caso México puede enunciarse cierta evidencia en los escritos de Ramírez (1996), Castillo y Herrera (2002), Fonseca (2009) y Ramírez y López (2015). En el primer caso se aporta evidencia sobre un crowding in para el periodo de estudio de 1950 a 1991, derivado del incremento en la productividad privada que tuvo el gasto público en infraestructura económica y social. Castillo y Herrera (2002) realizan un análisis 
del gasto de gobierno y del gasto privado para el periodo de 1980:1-2002:2, en el cual aportan evidencia sobre un efecto diferenciado en función de la temporalidad, en el corto plazo se da un efecto crowding out, mientras que en el largo plazo se presenta un efecto crowding in. A una conclusión similar llega Fonseca (2009), quien aporta evidencia de un desplazamiento parcial de la inversión pública hacia la inversión privada en el corto plazo, mientras que en el largo plazo encuentra un débil y marginal efecto de complementariedad entre la inversión pública y privada. Ramírez y López (2015) en un análisis sobre la formación bruta de capital público y privado correspondiente al periodo 1993:1-2013:4, observan un efecto de retroalimentación entre dichas variables y el crecimiento económico. Por su parte, Calderón y Roa (2006) aportan evidencia sobre el desplazamiento de la formación bruta de capital fijo por el gasto corriente del gobierno federal en el periodo 1983-2006.

Los efectos registrados en la economía mexicana son convergentes con lo observado en una gran cantidad de estudios empíricos para diversos países y en periodos temporales diferentes. Después de una amplia revisión del tema, Pereira y Andraz (2012) concluyen que existe cierto acuerdo en algunos casos, como el efecto positivo que tiene la inversión en infraestructura, el que dicho efecto sea sustancialmente más alto en países menos desarrollados y que la inversión pública afecte a la inversión privada en el largo plazo de forma distinta de acuerdo con la región y con el sector.

\section{I.3. La inversión y la composición sectorial}

Como se ha comentado, el efecto de la inversión pública sobre la privada, tomando en cuenta el sector de actividad económica, puede tener algunas repercusiones interesantes en el establecimiento de la política de inversión de un país. El planteamiento de la problemática podría llegar incluso a generar algunas disyuntivas relacionadas con la eficiencia en el uso de los recursos y la equidad en la distribución del ingreso y el desarrollo de los sectores.

De conformidad con la teoría de las economías industrializadas y desindustrializadas (Banco Mundial, 2012), es lógico pensar que en un inicio las economías empezaron con un sector primario muy importante en relación con otras actividades, pero a medida que el ingreso per cápita se incrementa, dicho sector va perdiendo participación. Es posible que esto se deba a un cambio en los patrones de consumo o bien al incremento de la productividad relativa de la mano de obra de los tres sectores. Ante un incremento del PIB per cápita la demanda por alimento tiende a estabilizarse y comienza la demanda de otro tipo de bienes no perecederos que son 
producidos por el sector secundario ${ }^{4}$. Conforme crece el ingreso, los bienes industrializados comienzan a tener menor crecimiento en su demanda ya que, en parte, son sustituidos por diversos servicios como educación, salud, entretenimiento, entre otros. Es por ello que de acuerdo con la evolución del nivel de ingresos per cápita de determinado país, se puede establecer la hipótesis de que, en dicho país, se experimenta un cambio en la estructura productiva, se va concentrando la actividad en el sector servicios como consecuencia de un incremento en la productividad de los otros dos sectores.

A medida que se desplazan recursos al sector servicios, el efecto que se tendrá sobre la economía en general dependerá de los servicios a los cuales se destinen los recursos ya que, de acuerdo con Eichengreen y Gupta (2009), en los países de bajos ingresos el incremento del sector servicios se da principalmente en servicios tradicionales con poco impacto, mientras que en los países de ingresos altos el sector crece con la ampliación de los servicios financieros, técnicos, legales, de publicidad y negocios, esto incide de mejor manera al resto de los sectores. En el caso de México, como en el de otros países considerados en vías de desarrollo, se ha experimentado una terciarización precoz y una acelerada desindustrialización con resultados poco satisfactorios (Calderón y Hernández, 2016).

El efecto que tiene la inversión pública sobre cada uno de los sectores puede ser diferente, tal como lo denotan Saeed et al. (2006), al desglosar la actividad económica de Pakistán en sectores de actividad tales como la agricultura, manufacturas y el complemento, encuentran que hay crowding in en agricultura, crowding out en manufacturas y en el complemento los resultados no son robustos, estadísticamente hablando. Con base en esos resultados, ellos sugieren que se debe ampliar la inversión pública en agricultura y reducirla en manufacturas, ya que con ello se tendrá una mayor eficiencia en la economía.

Para el caso de Japón, Annala et al. (2008), realizan un estudio en el cual se valoran los impactos de la inversión pública sobre el empleo, la producción y la inversión privada, en diferentes sectores, mediante la utilización de un modelo VAR/ ECM. En ese estudio se comprueba que la inversión pública no afecta a todos los sectores de la misma manera. En lo referente al empleo tiene efectos amplios en el sector público, en el sector financiero, de la construcción y manufacturero. La inversión pública tiene impactos positivos en la producción de sectores tales como la minería, el financiero, el comercio y las manufacturas. Por último, la inversión privada se ve afectada por la inversión pública en industrias dedicadas a las comunicaciones y transportes, el comercio y los servicios financieros. Por su parte,

${ }^{4}$ El efecto es conocido como la Ley de Engel. 
Fujii et al. (2013), argumentan que el efecto de la inversión pública sobre la privada en cada sector está influenciado por la elasticidad de la demanda de inversión respecto de la tasa de interés, se registra la presencia del efecto crowding out en los sectores del comercio, construcción, comunicaciones y transportes y del crowding in en los sectores de servicios públicos y agricultura.

En México la composición sectorial es distinta en relación con la región que se estudie y en ese sentido es posible que la inversión pública pueda tener impactos diferentes. Costa-i-Font y Rodriguez-Oreggia (2005) analizan el impacto distributivo que tiene la inversión pública en México entre regiones, categorizan las regiones en función de la composición de los sectores y establecen una preponderancia del sector servicios en la capital, el sector secundario tiene mayor presencia en el norte y algunas partes del centro del país, es menor en el sur; el sector primario tiene principal relevancia en el sur y este del país. El sur es la región de mayor pobreza y el este un corredor industrial importante. Esos autores realizaron una distinción entre la inversión pública tomando en cuenta la realizada en infraestructura y la relativa a la inversión social, dentro de sus principales conclusiones destaca que el mejoramiento del ingreso en las regiones ricas concentra el impacto de la inversión en infraestructura más que la social, llevando a dichas regiones a una mejor condición. Naturalmente, de lo anterior es destacable el impacto diferenciado de la inversión pública en función de las características previas de las regiones.

\section{Aspectos metodológicos}

El modelo auto-regresivo de rezagos distribuidos o ARDL, por Autoregressive Distribuited Lag, se ha utilizado desde hace casi 50 años para el análisis de series de tiempo uniecuacionales, su especificación puede representarse como

$$
\begin{gathered}
y_{t}=c_{0}+c_{1} t+\sum_{i=1}^{p} \phi_{i} y_{t-i}+\sum_{j=0}^{q} \beta_{j}^{\prime} \vec{x}_{t-j}+u_{t}, \\
t=\max (p, q), \ldots, T .
\end{gathered}
$$

Para simplificar esta exposición, se puede suponer que el orden del rezago, $q$, es igual para todas las variables que se encuentran contenidas en $\vec{x}_{t}$, un vector de dimensión $k \times 1$; de acuerdo con Pesaran, Shin y Smith (2001), tanto las variables contenidas en dicho vector como la propia variable dependiente pueden ser variables $I(0), I(1)$ o incluso ser variables cointegradas, sin embargo, no se considera la posibilidad de que sean procesos con raíces unitarias estacionales o con raíces explosivas. 
Por lo tanto, como lo proponen Pesaran y Shin (1998), este modelo proporciona un enfoque para abordar el análisis de las relaciones de largo plazo entre series de tiempo de variables económicas, empezando por someter a prueba la hipótesis nula de no cointegración probando si existe una relación estadísticamente significativa entre los niveles de las series. La popularidad del modelo ARDL se ha incrementado notoriamente debido a que, para la prueba de cointegración se incluye una reparametrización en forma de un modelo que incluye un mecanismo de corrección de errores (o corrección al equilibrio de largo plazo), dando lugar a la representación

$$
\Delta y_{t}=c_{0}+c_{1} t+\alpha\left(y_{t-1}-\vec{\theta} \vec{x}_{t-1}\right)+\sum_{i=1}^{p-1} \psi_{i} \Delta y_{t-i}+\vec{\omega}^{\prime} \Delta \vec{x}_{t}+\sum_{j=1}^{q-1} \vec{\varphi}^{\prime} \Delta \vec{x}_{t-j}+\xi_{t},
$$

donde el coeficiente de la velocidad del ajuste $\alpha=1-\sum_{i=1}^{p} \phi_{i}$ y los coeficientes de largo plazo $\theta_{k j}=\frac{\sum_{j=0}^{q} \beta_{k j}}{\alpha}$.

El enfoque propuesto por Pesaran, Shin y Smith (2001) para el análisis de cointegración considera cinco casos posibles en relación con los términos deterministas incluidos en la ecuación: $i$ ) no constante ni tendencia; ii) constante restringida, no tendencia; iii) constante no restringida, no tendencia; $i v$ ) constante no restringi$\mathrm{da}$, tendencia restringida; $\mathrm{y}$, por último, $v$ ) constante y tendencia no restringidas. Por lo tanto, el primer paso consiste en decidir sobre los términos deterministas que se incluirán en el modelo. Después se selecciona el orden óptimo de rezagos para las variables incluidas y se estima el modelo ARDL mediante mínimos cuadrados ordinarios. Con base en los resultados de la estimación se calcula el valor del estadístico

$F$ correspondiente a la hipótesis nula conjunta $H_{0}:(\alpha=0) \cap\left(\sum_{j=0}^{q} \beta_{j}=0\right)$, comparando el resultado con los valores críticos correspondientes; en caso de rechazar esta hipótesis, se calcula el valor del estadístico $t$ para la hipótesis nula singular $H_{0}: \alpha=0$ y se compara con sus valores críticos. Pesaran, Shin y Smith (2001) proporcionan cotas inferiores y superiores para los valores críticos asintóticos dependiendo del número de regresores, el orden de integración de los mismos y los términos deterministas incluidos en el modelo. Con base en dichos límites las decisiones en relación con los resultados de la prueba es que las hipótesis nulas no pueden rechazarse si el valor calculado del estadístico se encuentra más cercano a cero que la cota inferior, se rechazan si dicho valor es mayor que la cota superior; si ambas hipótesis se rechazan se considera confirmada la existencia de una relación de largo plazo entre las variables analizadas. 


\section{ANÁLISIS EMPÍRICO}

En el análisis que se presenta en esta sección se emplea el PIB de los sectores primario, secundario y terciario como representantes del desempeño económico en el nivel sectorial. Como representantes de la inversión pública y privada se utilizan los datos de la formación bruta de capital fijo, tanto público como privado. Como tasa de interés relevante se emplea la Tasa de Interés Interbancaria de Equilibrio (TIE) a 28 días. Todas las observaciones corresponden al valor final alcanzado al final de los trimestres comprendidos en el periodo que va desde el inicio de 1993 hasta fines de 2015. Los datos sobre el PIB sectorial, así como la formación bruta de capital fijo, tanto público como privado, y la TIIE a 28 días, se han tomado del sitio web del Banco de México.

En la figura 1 se muestran los niveles del PIB de los tres sectores, expresados en millones de pesos de 2008. En términos reales, el valor del producto de los sectores secundario y terciario ha superado notoriamente el correspondiente al valor de la producción del sector primario, de esta figura se puede deducir que la tasa de crecimiento del sector secundario es menor que la del terciario, razón por la cual se puede ver también una brecha creciente entre el producto de ambos sectores. Además, se puede observar la trayectoria visiblemente más errática del sector primario, se observan caídas durante 2005 y 2010-2011 que no se ven a simple vista en la dinámica de los otros sectores. No obstante, es conveniente señalar que hay también caídas que son coincidentes en el PIB de los tres sectores. El primer caso que puede detectarse a simple vista es la caída que se encuentra asociada con la crisis de 1994, sin embargo, es destacable que la caída del sector primario antecede en un trimestre a la baja observada en los otros dos sectores. En el caso de las caídas en el producto de los tres sectores, se encuentra la relacionada con el colapso financiero de 2008, durante el cual la caída del sector terciario se anticipa en un trimestre a las caídas observadas en los sectores primario y secundario. De acuerdo con el Banco de México en 2005 (Banxico, 2006), el nivel de actividad económica fue menor con respecto al año previo, tuvo sus principales debilidades en la primera mitad del año, además presentó un comportamiento no balanceado entre sectores, así, el sector primario registró una caída y una amplia volatilidad, mientras que el sector secundario tuvo un resultado moderado, sustentado principalmente en la producción manufacturera, el sector terciario por su parte tuvo una expansión significativa. La caída y volatilidad del sector primario fue explicada por Banxico como consecuencia de las condiciones climáticas adversas que se presentaron durante dicho año. En el segundo semestre de 2011 se observó una desaceleración en la actividad económica que se mantuvo a lo largo del año con menor intensidad, según Banxico (2012), fueron las condiciones climáticas, otra vez, las causas de una contracción en el sector primario, mientras que las condiciones a nivel internacional, la situación financiera de los países de la zona euro y la desaceleración de la producción industrial en 
Estados Unidos, tuvieron impactos negativos en el sector secundario. En contraste con lo anterior, el sector terciario registró una trayectoria positiva mostrando signos de desaceleración sólo en el último trimestre de ese año

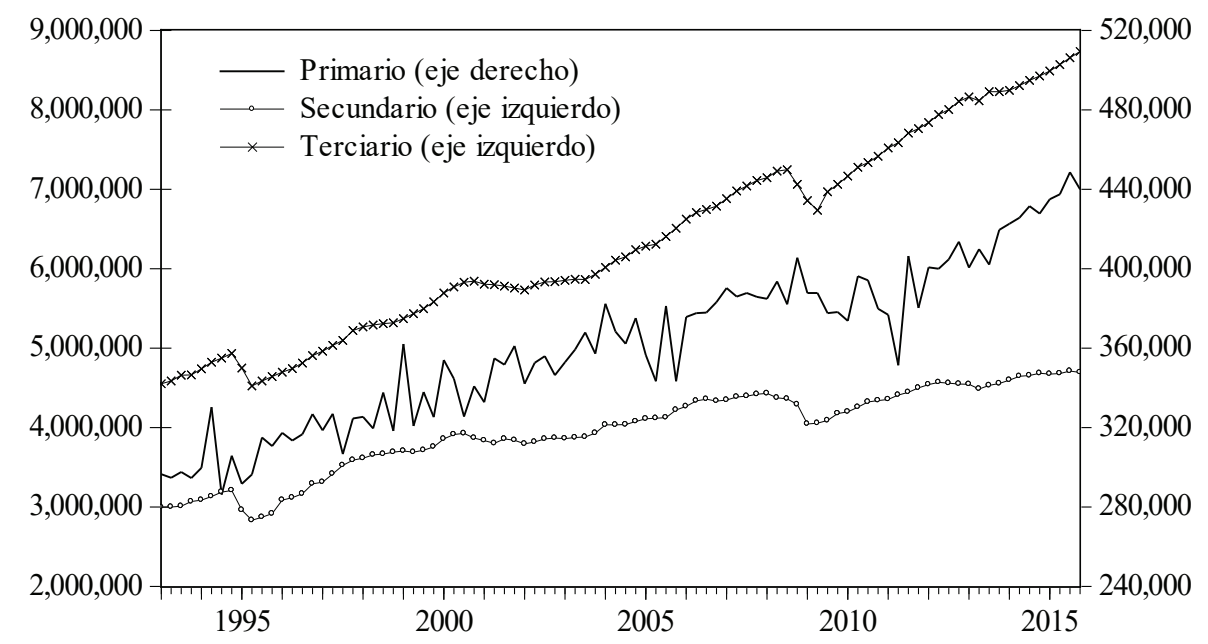

Figura 1. Producto sectorial real en México 1993-2015.

(Millones de pesos de 2008)

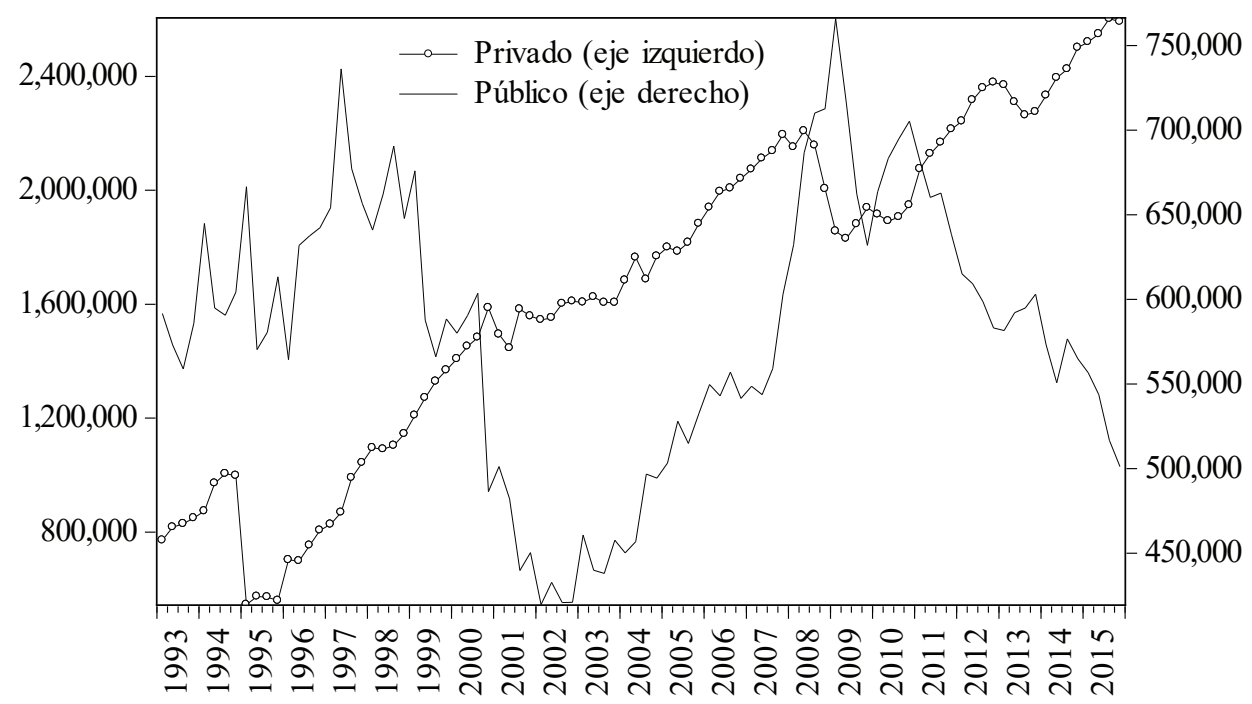

Figura 2. Formación bruta de capital fijo real en México 1993-2015.

(millones de pesos de 2008) 
La figura 2 muestra el comportamiento de la inversión privada y pública en términos reales. A simple vista destaca que entre 1993 y 2015 el comportamiento de la inversión pública, además de ser de menor cuantía, ha sido mucho más errático que el observado en la inversión privada. Es ostensible que la caída, que se ha dado en forma sostenida a partir de principios de 2009, ha llevado a que, a fines de 2015 la inversión pública se encuentre en niveles similares a los observados a fines de 2005, periodo en el que venía recuperándose de los mínimos observados en 2002. Por otra parte, a pesar de su tendencia creciente en el tiempo, en la inversión privada se observan caídas importantes, las cuales pueden asociarse a la crisis mexicana gestada durante 1994 y la crisis mundial desatada en 2008; también pueden observarse caídas de menor escala como las ocurridas a principio de 2001 e inicios de 2013. Como se señaló, la inversión pública muestra una dinámica con caídas más abruptas, siendo claramente notables las caídas observadas a fines de 2000 y de 2009. Cabe destacar que de acuerdo con las tablas de origen-destino de la formación bruta de capital fijo publicadas por el Instituto Nacional de Estadística y Geografía (INEGI) ${ }^{5}$, la inversión destinada a las actividades primarias en relación con el total pasó de 3.3 por ciento a 1.5 por ciento de 2003 a 2015, en una clara tendencia a la baja, mientras que el sector terciario acapara cerca de dos tercios del total de la inversión pública y privada.

En la figura 3 se observa el comportamiento de la TIIE real a 28 días, se observa el gran salto dado a principios de 1995, claramente relacionado con la crisis del peso mexicano que detona la posterior crisis económica. Posteriormente únicamente se observa un alza importante, que puede asociarse fácilmente con la volatilidad financiera mundial consecuencia de la crisis rusa de 1998. Se puede observar que en los últimos años la TIIE ha tenido una dinámica relativamente estable en torno a un valor de cero, incluso negativa en algunos trimestres, como lo fue después de la crisis de 1994 e inicios de 1997.

Para proseguir este análisis empírico del desempeño de los sectores, se procedió a llevar a cabo pruebas de raíces unitarias para determinar los grados de integración de las series bajo estudio, los resultados se presentan en el cuadro 1. Con excepción de la TIIE, el análisis de la estacionariedad de las demás variables se llevó a cabo sobre los logaritmos de los valores observados. Sólo en el caso de la TIIE las pruebas con diferentes especificaciones de los términos deterministas permitieron rechazar la hipótesis nula de no estacionariedad, en los demás casos se tuvo que recurrir a las primeras diferencias de los logaritmos de las series originales para poder rechazar la hipótesis nula, esto sugiere que el orden de integración

\footnotetext{
${ }^{5}$ www.inegi.org.mx/est/contenidos/proyectos/cn/fbkf.
} 
de las series originales así como de sus logaritmos es $I(1)$. La prueba de los logaritmos del PIB terciario considerando un término constante y una tendencia, sugirieron el rechazo de la hipótesis nula incluso al 5 por ciento de significancia, sin embargo, las otras especificaciones de la prueba y la inspección visual de la figura 1 sugieren que dicha variable no muestra un comportamiento estacionario.

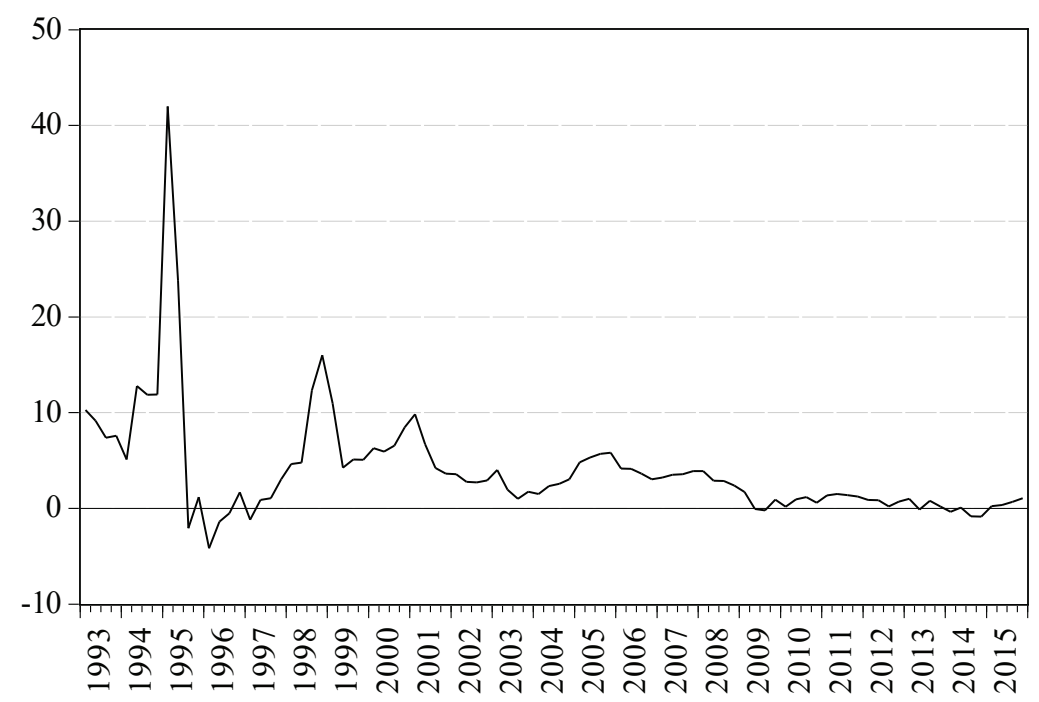

Figura 3. Tasa de interés interbancaria de equilibrio real en México 1993-2015 (\%)

Cuadro 1. Pruebas de raíz unitaria Dickey Fuller Aumentadas

\begin{tabular}{|l|l|l|l|l|l|l|}
\hline \multirow{2}{*}{ VARIABLE } & \multicolumn{5}{|c|}{ TÉRMINOS DETERMINISTAS } \\
\hline & \multicolumn{2}{|c|}{ NINGUNO } & \multicolumn{2}{c|}{ CONSTANTE } & \multicolumn{2}{c|}{ CONSTANTE Y TENDENCIA } \\
\hline & NIVEL & $\begin{array}{c}\text { PRIMERA } \\
\text { DIFERENCIA }\end{array}$ & \multicolumn{2}{|c|}{ NIVEL } & $\begin{array}{c}\text { PRIMERA } \\
\text { DIFERENCIA }\end{array}$ & \multicolumn{2}{c|}{ NIVEL } & $\begin{array}{c}\text { PRIMERA } \\
\text { DIFERENCIA }\end{array}$ \\
\hline primario & 1.976202 & $-10.367940^{* * *}$ & -0.405181 & $-7.681174^{* * *}$ & -2.686162 & $-7.635474^{* * *}$ \\
\hline secundario & 1.928450 & $-4.695673^{* * *}$ & -1.549483 & $-6.389838^{* * *}$ & -2.518392 & $-6.414577^{* * *}$ \\
\hline terciario & 3.081798 & $-4.811976^{* * *}$ & -0.239230 & $-5.907218^{* * *}$ & $-3.998247^{* *}$ & $-5.879241^{* * *}$ \\
\hline fbcfpriv & 1.474893 & $-4.385086^{* * *}$ & -0.770351 & $-5.208923^{* * *}$ & -2.182592 & $-9.229535^{* * *}$ \\
\hline fbcfpub & -0.545180 & $-4.386494^{* * *}$ & -2.879992 & $-4.378749^{* * *}$ & -2.836950 & $-4.352501^{* * *}$ \\
\hline tiie & $-3.764403^{* * *}$ & $-6.149804^{* * *}$ & $-3.380302^{* *}$ & $-6.118227^{* * *}$ & $-4.195737^{* * *}$ & $-6.082031^{* * *}$ \\
\hline
\end{tabular}

Las pruebas de raíz unitaria se hicieron para los logaritmos de las series analizadas, con excepción de la tiie la cual se encuentra expresada en tanto por uno.

$* * *, * *, *$ Denotan, respectivamente, 1 por ciento, 5 por ciento y 10 por ciento de significancia. 
142 ECONOMÍA TeORÍA Y PRÁCTICA • Nueva Época, número 48, enero-junio 2018

Cuadro 2. Prueba de cointegración de Johansen

\begin{tabular}{|c|c|c|}
\hline \multicolumn{3}{|c|}{ SERIES INCLUIDAS EN EL ANÁLISIS: PRIMARIO, SECUNDARIO, } \\
\hline$H_{0}:$ RELACIONES COINTEGRANES & TRAZA & $\begin{array}{c}\text { VALOR } \\
\text { PROPIO }\end{array}$ \\
\hline 0 & $109.37220^{* * *}$ & $51.034560^{* * *}$ \\
\hline$\leq_{1}$ & 58.33769 & 26.06369 \\
\hline$\leq_{2}$ & 32.27400 & 16.73493 \\
\hline$\leq 3$ & 15.53907 & 12.19685 \\
\hline$\leq 4$ & 3.342225 & 2.528053 \\
\hline$\leq 5$ & 0.814172 & 0.814172 \\
\hline
\end{tabular}

Con base en los resultados antes indicados respecto de la estacionariedad de las series, se llevaron a cabo las pruebas de cointegración de Johansen, cuyos resultados, en el cuadro 2, muestran que tanto la prueba de la traza como la del valor propio máximo sugieren rechazar la hipótesis de que no existen relaciones cointegrantes, ambas pruebas también muestran, que no se puede rechazar la existencia de una relación cointegrante a lo más. Por lo anterior, se puede concluir que las series analizadas se encuentran cointegradas y que entre ellas existe una relación de largo plazo.

En el cuadro 3 se puede ver que los resultados de las pruebas $F$ y $t$ de Pesaran, Shin y Smith (2001) aportan evidencia de que el desempeño económico del sector primario mantiene una relación de largo plazo con el desempeño de los sectores secundario y terciario, así como con la tasa de interés y la inversión pública y privada. De acuerdo con la magnitud del coeficiente de velocidad del ajuste (-0.8407), un desequilibrio del sector tardará poco más de un trimestre en corregirse. Los coeficientes de ajuste de los sectores secundario y terciario son también significativos, el del secundario al 5 por ciento, de menor magnitud en comparación con el del primario, implica tiempos de ajuste de aproximadamente dos años y medio y dos años, respectivamente.

El desempeño de los sectores secundario y terciario impacta positivamente al sector primario, lo que puede explicarse dado que el crecimiento en aquellos implica la adquisición de materias primas como insumos para las actividades productivas y comerciales; esta inferencia se corrobora con los coeficientes correspondientes estimados en la dinámica de la ecuación. Dichas actividades pueden mejorar a su 
Cuadro 3. Modelos ARDL estimados como mecanismos de corrección de errores

\begin{tabular}{|c|c|c|c|c|c|c|c|c|}
\hline \multicolumn{3}{|c|}{$\Delta$ primario $_{t}$} & \multicolumn{3}{|c|}{$\Delta$ secundario $_{t}$} & \multicolumn{3}{|c|}{$\Delta$ terciario $_{t}$} \\
\hline$\alpha$ & -0.8407 & $* * *$ & $\alpha$ & -0.0940 & ** & $\alpha$ & -0.1220 & $\star \star \star *$ \\
\hline secundario $_{\mathrm{t}}$ & 0.6369 & $* * *$ & primario $_{t-1}$ & -0.5057 & & primario $_{t-1}$ & 0.3288 & $* *$ \\
\hline terciario ${ }_{t-1}$ & 0.5705 & $* * *$ & terciario ${ }_{t-1}$ & 0.4684 & & secundario ${ }_{t-1}$ & -0.4199 & \\
\hline fbcfpriv $_{t-1}$ & -0.2329 & $* * *$ & fbcfpriv $t_{t-1}$ & 0.0863 & & fbcfpriv $v_{t-1}$ & 0.2671 & $* * *$ \\
\hline$f b c f p u b_{t-1}$ & -0.1868 & $* * *$ & fbcfpub $_{t-1}$ & -0.0770 & & fbcfpub $_{t-1}$ & 0.1780 & $* * *$ \\
\hline tiie $_{t-1}$ & -0.2422 & $* * *$ & tiie $_{t-1}$ & -0.8090 & & $\mathrm{tiie}_{\mathrm{t}-1}$ & 0.4795 & * \\
\hline$\Delta$ primario $_{t-1}$ & -0.3925 & $* * *$ & $\Delta$ secundario $_{t-1}$ & -0.1889 & $* *$ & tendencia & 0.0053 & $* * *$ \\
\hline$\Delta$ primario $_{t-2}$ & -0.1576 & $* *$ & $\Delta$ primario $_{t}$ & 0.0575 & ** & $\Delta$ primario $_{t}$ & 0.0401 & ** \\
\hline$\Delta$ secundario $_{t}$ & 0.53548 & $* * *$ & $\Delta$ primario $_{t-1}$ & 0.1482 & $* * *$ & $\Delta$ secundario $_{t}$ & 0.2224 & $* * *$ \\
\hline$\Delta$ terciario $_{t}$ & 0.4797 & $* * *$ & $\Delta$ primario $_{t-2}$ & 0.0965 & $* * *$ & $\Delta$ fbcfpriv $_{t}$ & 0.0965 & $* * *$ \\
\hline$\Delta$ fbcfpriv $t_{t}$ & -0.1958 & $* * *$ & $\Delta$ terciario $_{t}$ & 0.6014 & $* * *$ & $\Delta$ fbcfpriv $v_{t-1}$ & 0.0468 & $* * *$ \\
\hline$\Delta f b c f p u b_{t}$ & -0.0838 & * & $\Delta$ terciario $_{t-1}$ & 0.4078 & $* * *$ & $\Delta$ fbcfpriv $_{t-2}$ & 0.0478 & $* * *$ \\
\hline$\Delta$ fbcfpub $_{t-1}$ & 0.0723 & & $\Delta$ fbcfpriv $_{t}$ & 0.1355 & $* * *$ & $\Delta f b c f p u b_{t}$ & 0.0425 & $* * *$ \\
\hline$\Delta$ tiie $_{t}$ & -0.2036 & $* * *$ & $\Delta$ fbcfpriv $_{t-1}$ & -0.0203 & & $\Delta$ tiie $_{t}$ & -0.0086 & \\
\hline 1994:2 & 0.1261 & $* * *$ & $\Delta f b c f p r i v_{t-2}$ & -0.0616 & $* * *$ & $\Delta t i i e_{t-1}$ & -0.0662 & $* * *$ \\
\hline 1999:1 & 0.1095 & $* * *$ & $\Delta$ fbcfpriv $v_{t-3}$ & 0.0272 & *** & $\Delta t_{i i i_{t-2}}$ & 0.0345 & ** \\
\hline 2005:2 & -0.0760 & $* * *$ & $\Delta f b c f p u b_{t}$ & 0.0223 & & 1995:1 & 0.0347 & ** \\
\hline 2008:4 & 0.0758 & $* * *$ & $\Delta$ tiie $_{t}$ & 0.0734 & ** & 1999:1 & -0.0130 & $* *$ \\
\hline 2009:1 & 0.0857 & $* * *$ & $1994: 2$ & -0.0345 & $* * *$ & $2008: 4$ & -0.0258 & *** \\
\hline 2009:2 & 0.0614 & ** & $2005: 2$ & 0.0138 & ** & $2009: 1$ & -0.0187 & $* * *$ \\
\hline \multirow[t]{5}{*}{ 2011:2 } & -0.1103 & $* * *$ & $2008: 4$ & 0.0160 & * & $2009: 2$ & -0.0244 & $* * *$ \\
\hline & & & 2009:1 & -0.0207 & ** & 2009:3 & 0.0206 & *** \\
\hline & & & $2009: 2$ & 0.0191 & * & constante & 1.3068 & $* *$ \\
\hline & & & $2011: 2$ & 0.0153 & ** & & & \\
\hline & & & constante & 1.3266 & *** & & & \\
\hline$F(\mathrm{PSS})$ & 15.711 & $* * *$ & & 9.324 & $* * *$ & & 5.002 & $* * *$ \\
\hline$t(\mathrm{PSS})$ & -7.731 & $* * *$ & & -2.230 & & & n.d. & \\
\hline $\mathrm{R}^{2}$ & \multicolumn{2}{|l|}{0.8386} & & \multicolumn{2}{|l|}{0.9177} & & \multicolumn{2}{|l|}{0.9036} \\
\hline Log-verosimilitud & \multicolumn{2}{|l|}{220.428} & & \multicolumn{2}{|l|}{343.991} & & \multicolumn{2}{|l|}{368.698} \\
\hline Akaike & \multicolumn{2}{|l|}{-406.857} & & \multicolumn{2}{|l|}{$\begin{array}{l}-637.982 \\
\end{array}$} & & \multicolumn{2}{|l|}{$\begin{array}{l}-693.387 \\
\end{array}$} \\
\hline Schwartz & \multicolumn{2}{|l|}{-364.742} & & \multicolumn{2}{|l|}{-576.049} & & \multicolumn{2}{|l|}{-638.886} \\
\hline \multicolumn{9}{|c|}{$\begin{array}{l}* * *, * * \text { y } \text { denotan, respectivamente, el } 1 \text { por ciento, } 5 \text { por ciento y } 10 \text { por ciento de significancia. } \\
F \text { (PSS) y } t \text { (PSS) son los estadísticos de Pesaran, Shin y Smith (2001) con los valores críticos al } 1 \text { por } \\
\text { ciento de significancia; sin términos deterministas para el sector primario, incluyendo una constante } \\
\text { no restringida para el sector secundario y con una constante no restringida y tendencia restringida } \\
\text { para el sector terciario } \\
\text { n.d. = no disponible }\end{array}$} \\
\hline
\end{tabular}


vez el desempeño del sector primario al dotarlo de herramientas, maquinarias, fertilizantes, entre otros implementos, y de servicios tales como el transporte, servicios públicos y redes de distribución. En primera instancia puede parecer paradójico que el producto del sector primario se relacione negativamente con la inversión, tanto pública como privada. Sin embargo, es sabido que la dinámica del sector ha perdido participación en favor de actividades de los otros sectores debido a los procesos de urbanización con el consecuente crecimiento del sector servicios acompañado por la creación de infraestructura de comunicaciones y transporte entre los centros urbanos. Adicionalmente, se podría considerar que la inversión pudiera no ser suficiente ni oportuna, además de la posibilidad de que se invierta como respuesta a fenómenos climatológicos que afectan al sector en forma normal y no para incrementar su productividad. La tasa de interés afecta en forma negativa al sector sugiriendo que, a pesar de que ésta ha caído en términos reales, como se observa en la figura 3 , sus incrementos encarecen el costo del financiamiento y afectan negativamente el desempeño del sector.

Por otra parte, el producto de los otros sectores, la inversión (pública y privada) y la tasa de interés no muestran efectos estadísticamente significativos sobre el comportamiento del sector secundario en el largo plazo; sin embargo, en el corto plazo existen vinculaciones significativas y con signo positivo. La inversión privada muestra efectos significativos sobre el producto del sector, predominan los que tienen signo positivo. Por su parte, la inversión pública no muestra efecto alguno. En cuanto a la tasa de interés se observa que su dinámica ha contribuido a elevar el nivel del producto.

En cuanto al sector terciario, a pesar de que el coeficiente asociado con el sector secundario no es significativo, se muestra evidencia de que sí existe una relación de largo plazo que lo lleva a corregir un desequilibrio respecto de esa relación en aproximadamente dos años. La inversión pública y privada han ejercido una influencia positiva en los niveles de producto del sector terciario, esto sugiere que la inversión pública y privada presentan el efecto crowding in y contribuyen simultáneamente a un mejor desempeño. La tasa de interés real también resulta significativa para explicar el comportamiento del sector, sin embargo sus efectos son mixtos. Por un lado se observa un signo negativo que puede ser congruente con el hecho de que las alzas en la tasa de interés conllevan a un encarecimiento del crédito y a un menor desempeño por los egresos asociados y la desincentivación de la actividad del sector. Por otra parte, dado que los egresos por financiamiento se quedan en la esfera del sector terciario, se podría entender el signo positivo observado.

Las variables dummies asociadas con periodos en que ocurrieron eventos específicos muestran efectos diferenciados en los sectores. En el segundo trimestre 
de 1994 se observa un signo positivo para el sector primario, probablemente debido a la derrama económica asociada con el proceso electoral federal, en tanto que para el sector secundario se observa un signo negativo, posiblemente explicado por el enturbiamiento de las expectativas, por el asesinato del candidato presidencial priista y el deterioro de las reservas internacionales. Al parecer el inicio de la crisis de 1995 favoreció en términos reales el comportamiento del sector de los servicios, posiblemente debido a la sustitución de compras de productos importados por productos nacionales. El regreso de la tasa de interés real de dos dígitos a uno después de la crisis financiera rusa y de sus efectos en los mercados mundiales, pudo haber impulsado la actividad en el sector primario al mismo tiempo que produjo una baja importante de ingresos en la parte financiera del sector servicios. Tanto la variable dummy asociada con los eventos de 2005 como la relacionada con los de 2011 resultaron significativas con el signo que cabría esperar. El periodo de la crisis subprime muestra efectos en todos los sectores, positivos en el primario y negativos en los otros dos. En el caso del sector primario podría pensarse que ocurrió un desplazamiento de la demanda de productos básicos (principalmente alimentos y materias primas) en favor de la oferta nacional. Parece obvio que las caídas de los sectores secundario y terciario, dada su importancia en el PIB, explican la caída general de la economía mexicana.

\section{ConCLUSIONES}

En este trabajo se han analizado las relaciones entre el comportamiento y los sectores de actividad económica en México, así como los efectos de la inversión pública, la inversión privada y la tasa de interés sobre el desempeño sectorial. A pesar de que se observan dinámicas sectoriales diferentes durante el periodo del análisis, los tres sectores responden de manera significativa a la relación de largo plazo, ajustándose a diferentes velocidades, el sector primario es el de más rápido ajuste, en tanto que el sector secundario lo hace notoriamente en forma más lenta. Esta diferencia en tiempos de ajustes parece obedecer a que el desempeño del sector secundario está sujeto a una lógica diferente a las de los otros sectores, quizá en parte explicable por una mayor vinculación con el sector externo como lo sugiere Banxico. Entre los aspectos obvios que hacen diferente el comportamiento de los sectores se encuentra la vulnerabilidad mostrada por el sector primario, dada su dependencia de las condiciones climáticas, así como la baja y decreciente inversión. Naturalmente, el hecho de que el grueso de la inversión se concentre en el sector terciario, tiene como efecto que su desempeño se vea favorecido por ella, tanto en el largo como en el corto plazo. La dinámica de la tasa de interés no parece adecuada para favorecer el desempeño del sector 
primario ni en el corto ni en el largo plazo, más bien lo que se puede decir es que ha resultado contraproducente en términos reales. El comportamiento de la tasa de interés en el largo plazo no ha tenido consecuencia significativa para el sector secundario, tiene efectos positivos únicamente en la dinámica de corto plazo. Para el desempeño del sector terciario en el largo plazo, la tasa de interés le ha favorecido aunque en forma no tan significativa, encontramos efectos mixtos en la dinámica de corto plazo, esto puede explicarse debido a los beneficios que los movimientos de la tasa de interés en términos reales pueden generar en los diferentes subsectores.

Evidentemente, la política económica tiene mucho que hacer para lograr un desempeño sectorial más armónico y congruente con las necesidades de crecimiento económico. En el caso del sector primario resulta indispensable la ampliación de la inversión, así como el desarrollo de programas de financiamiento con tasas de interés acordes con el riesgo y los rendimientos del sector, además de la difusión de coberturas contra eventos climáticos para fomentar el incremento en su uso. Para el sector secundario parece ser necesario retomar una política industrial integral que vincule la inversión y los costos de financiamiento con el desempeño del sector y de la economía en su conjunto, de tal forma que se generen condiciones para el encadenamiento productivo intra e inter sectorial.

La agenda de investigación respectiva debería incluir estudios sobre el potencial y requerimientos para la integración del sector primario con el secundario mediante la generación de agroindustrias, identificando las actividades o enclaves de mayor impacto favorable. Por otra parte, también se requiere seguir investigando sobre la dinámica del sector secundario y su vinculación con las actividades de los otros sectores, de forma tal que se comprenda mejor su posible papel de impulsor y conector de dichas actividades.

\section{REFERENCIAS BibLIOGRÁFICAS}

Afonso, Antonio y Tovar, Joao (2015), "How does fiscal policy affect investment? evidence from a large panel”, Int. J. Fin. Econ., 20 (4), pp. 310-327.

Afonso, Antonio y Aubyn, Miguel (2009), "Macroeconomic Rates of Return of Public and Private Investment: Crowding-In and Crowding-Out Effects", The Manchester School, 77 (s1), pp. 21-39.

Ahmed, Habib y Miller, Stephen (2000), "Crowding-out and crowding-in Effects of the Components of Government Expenditure", Contemporary Economic Policy, 18 (1), pp. 124-133. 
Annala, Christopher; Batina, Raymond y Feehan, James (2008), "Empirical Impact of Public Infrastructure on the Japanese Economy", The Japanese Economic Review, 59 (4), pp. 419-437.

Banco Mundial (2002), "Growth of the Service Sector", Beyond Economic Growth, Banco Mundial.

Banxico (2006), Informe anual 2005, Banco de México. (2012), Informe anual 2011, Banco de México.

Barro, Robert (1974), “Are Government Bonds Net Wealth?”, The Journal of Political Economy, 82 (6), pp. 1095-1117. (1989), "The Ricardian Approach to Budget Deficit", Journal of Economic Perspectives, 3 (2), pp. 37-54.

(1996), "Reflections on Ricardian Equivalence", NBER, Working Paper 5502.

Bayraktar, Nihal y Moreno-Dodson, Blanca (2015), "How can public spending help you grow? An empirical analysis for developing countries", Bulletin of Economic Research, 67 (1), pp. 30-64.

Becker, Bettina (2015), "Public R\&D policies and private R\&D investment: a survey of the empirical evidence", Journal of Economic Surveys, 29 (5), pp. 917-942.

Beteta, Hugo E. y Moreno-Brid, Juan C. (2014), Cambio estructural y crecimiento en Centroamérica y la República Dominicana. Un balance de dos décadas, 1990-2011, Chile, CEPAL, 400 pp.

Bleaney, Michael; Gemmell, Norman y Kneller, Richard (2001), "Testing the Endogenous Growth Model: Public Expenditure, Taxation and Growth over the Long-Run", Canadian Journal of Economic, 34 (1), pp. 36-57.

Calderón, Cuauhtémoc y Roa, Rubén (2006), “¿Existe un crowding out del financiamiento privado en México?”, Análisis Económico, 2 (48), pp 139-150.

Calderón, Cuauhtémoc y Hernández, Leticia (2016), “Cambio estructural y desindustrialización en México", Panorama Económico, XII (23), pp. 153-190.

Carranza, Luis; Daude, Christian y Melguizo, Ángel (2014), "Public Infrastructure Investment and Fiscal Sustainability in Latin America: Incompatible Goals?", Journal of Economic Studies, 41 (1), pp. 29-50.

Castillo, Ramón y Herrera, Jorge (2005), “Efectos del gasto público sobre el gasto privado en México", Estudios Económicos, 20 (2), pp. 173-196.

Catao, Luis y Terrones, Marco (2003), "Fiscal Deficits and Inflation", IMF, Working Paper, 65. 
Costa-i-Font, Joan y Rodriguez-Oreggia, Eduardo (2005), "Is the Impact of Public Investment Neutral across the Regional Income Distribution? Evidence from Mexico", Economic Geography, 81 (3), pp. 305-322.

Eichengreen, Barry y Poonam, Gupta. (2009), "The Two Waves of Service Sector Growth", NBER, Working Paper 14968.

Eisner, Robert (1992), “Deficits: Which, How Much, and So What?", The American Economic Review, 82 (2), pp. 295-298.

Erden, Lutfi y Holcombe, Randall (2006), "The Linkage between Public and Private Investment: A Co-Integration Analysis of a Panel of Developing Countries", Eastern Economic Journal, 32 (3), pp. 479-492.

Fay, Marianne y Morrison, Mary (2007), Infraestructura en América Latina y el Caribe Acontecimientos recientes y desafios principales, Colombia, Banco Mundial, Mayol Ediciones S.A., p. 134.

Fonseca, Carlos (2001), "Ricardian equivalence: an empirical application to the Portuguese economy”, CES Discussion Paper, Series 12, Catholic University of Leuven.

Fonseca, Felipe. (2009), "El impacto de la inversión pública sobre la inversión privada en México, 1980-2007”, Estudios Económicos, 24 (2), pp. 187-224.

Fujii, Takao; Kazuki, Hiraga y Masafumi, kozuca (2013), "Effects of public investment on sectoral private investment: A factor augmented vAR approach", J. Japanese Int. Economies, 27, pp. 35-47.

Furceri, Davide y Sousa, Ricardo (2011), "The Impact of Government Spending on the Private Sector: Crowding-out versus Crowding-in Effects", Kyklos, International Review for Social Sciences, 64 (4), pp. 516-533.

Fuster, M. Luisa (1993), “La Hipótesis de Equivalencia Ricardiana: Un análisis empírico en los países de la Comunidad Europea", Investigaciones Económicas, XVII (3), pp. 495-506.

Haque, M. Emranul y Kneller, Richard (2015), "Why does public investment fail to raise economic growth? the role of corruption", The Manchester School, 83 (6), pp. 623-651.

Hirschman, Albert (1961), La estrategia del desarrollo económico, México, FCE, p. 211.

Kneller, Richard; Bleaney, Michael y Gemmell, Norman (1998), "Growth, Public Policy and Government Budget Constraint: Evidence from OECD Countries", Discussion Papers 14, School of Economics, University of Nottingham. Lasa, Jose (1997), Deuda, inflación y déficit. Una perspectiva macroeconómica de la política fiscal, México, UAM-I, p. 235. 
Lücke, Bernd (1998), "Econometric Tests of Ricardian Equivalence: Result for Germany", FinanzArchiv / Public Finance Analysis, 55 (3), pp. 288-314. Pereira, Alfredo y Andraz, Jorge (2012), "On the economic effects of public infrastructure investment: A survey of the international evidence", $C E F A G E-$ UE Working Paper 10.

Pereira, Alfredo (2000), "Is All Public Capital Created Equal?", The Review of Economics and Statistics, 82 (3), pp. 513-518.

Pesaran, M. Hashem y Shin, Yongcheol (1998), “An autoregressive distributed-lag modelling approach to cointegration analysis", Econometrics and Economic Theory in the 20 $0^{\text {th }}$ Century: The Ragnar Frisch Centennial Symposium, S. Strøm, Cambridge, Cambridge University Press, pp. 371-413.

Pesaran, M. Hashem; Shin, Yongcheol, y Smith, Richard (2001), "Bounds testing approaches to the analysis of level relationships", Journal of Applied Econometrics, 16 (3), pp. 289-326.

Posadas, Carlos (2008), "La economía y la economía política del atraso y del desatraso: notas sobre La estrategia del desarrollo económico de Albert Hirschman", Revista Desarrollo y Sociedad, 62, pp. 89-116.

Pritchett, Lant (2000), "The Tyranny of Concepts: CudiE (Cumulated, Depreciated, Investment Effort) Is Not Capital", Journal of Economic Growth, 5, pp. 361-384.

Ramírez, Eduardo y López-Herrera, Francisco (2014), “Inversión pública y privada en México y su incidencia en el crecimiento", Panorama Económico, 10 (19), pp. 53-76.

Ramírez, Eduardo (2007), "La contracción del gasto público y su poca viabilidad para lograr la disciplina fiscal", Comercio Exterior, Bancomext, 57 (2), pp. 99-113.

Ramírez, Miguel (1996), "Public and Private Investment in Mexico and Chile: An Empirical Test of the Complementarity Hypothesis", AEJ, 24 (4), pp. 301320 .

Ricciuti, Roberto (2003), “Assessing Ricardian Equivalence”, Journal of Economic Survey, 17 (1), pp. 55-78.

Saeed, Norman; Kalim, Hyder y Ahmad, Eatzaz (2006), “The Impact of Public Investment on Private Investment: A Disaggregated Analysis", The Pakistan Development Review, 45 (4), pp. 639-663.

Sargent, Thomas y Wallace, Neil (1981), "Some Unpleasent Monetarist Aritmetic”, Federal Reserve Bank of Minneapolis, Quarterly Review, 5 (3), pp. 1-17. 
150 ECONOMÍA TeORÍA Y PRÁCTICA • Nueva Época, número 48, enero-junio 2018

Traum, Nora y Yang, Shu-Chun (2015), "When Does Government Debt Crowd out Investment?", Journal of Applied Econometrics, 30 (1), pp. 24-45.

Vickrey, William (2000), "Fiteen Fatal Fallacies of Financial Fundamentalism-.A disquisition on Demand Side Economies", Working Paper 1, Center for Full Employment and Price Stability, University of Missouri-Kansas City. 